\title{
Direction of Arrival (DOA) Estimation in an Array of Antennas using Derivative-Free Methods
}

\author{
Raees M. Asif \\ Department of \\ Electrical/Telecom ICT \\ Islamabad affiliated with UET \\ Peshawar
}

\begin{abstract}
In this paper, direction of arrival estimation has been addressed using derivative free methods and their hybrid procedure in antenna array signal processing. The problem has been formulated in the root mean square error sense by considering the single snapshot. A scenario based simulation has been performed by taking different number of antenna elements with varying sources. The stability, reliability and applicability of the proposed scheme has been monitored by Monte Carlo simulation and their statistical analysis based on the fitness of the problem, global mean square error and computational budget in term of time complexity.
\end{abstract}

\section{General Terms}

Direction of arrival (DOA) estimation

\section{Keywords}

Array of antennas (ULA); Derivative free methods; Monte Carlo simulation; Genetic algorithm (GA); Pattern Search (PS); Genetic algorithm hybridized with pattern Search (GAPS)

\section{INTRODUCTION}

Direction of arrival estimation of impinging electromagnetic waves on an array of antennas has been important problem in many fields such as geo-physics and mobile communication, radar, radio astronomy and so on. DOA estimation techniques are used in position location and tracking system by many agencies. Highly accurate DOA estimation provides many advantages such as increasing the capacity, reducing the transmitting power of mobile terminal, resisting multipath effects, decreasing interference from outside [1].

Array based direction of Arrival (DOA) estimation techniques can be divided into four different types: Conventional techniques subspace based techniques, Maximum likelihood techniques, and integrated techniques. Conventional methods are based on classical beam forming techniques and require large number of elements to achieve high resolution. The very basic conventional narrowband DOA estimation method is beam former and Capon's minimum variance distortion less response method. (MVDR) is a super resolution method which means that its ability to resolve sources is better than that of beam former [9]. Beam formers are spatial filters used to suppress interferes and noise while the desired signal is maintained. Subspace based methods are high resolution suboptimal narrowband DOA techniques which exploit the Eigen structure of input data matrix and they are MUSIC, presented in [2], [7] and ESPRIT. MUSIC is a technique based on exploiting the Eigen structure of input covariance matrix. Eigen vectors are obtained by either an Eigen decomposition of sample covariance matrix or singular value decomposition (SVD) [3]. ESPRIT (Estimation of signal parameters via rotational invariant technique) dramatically reduces the computational and storage requirement of MUSIC.

\author{
Junaid Ali Khan \\ Hamdard Institute of \\ Engineering \& Technology, \\ Islamabad Campus
}

Maximum likelihood techniques are optimal techniques which can even perform well under low SNR conditions but are computationally very complex. Maximum likelihood techniques can perform well in coherent signal conditions as well [4], [5]. Integrated approach which is a combination of property restoral method and subspace-based techniques can separate multiple signals and estimate their direction of arrivals. The Iterative Least Squares Projection Based CMA (ILSP-CMA) is a property restoral based algorithm which can be used to detect the waveforms associated with multiple sources incident on a receiver array. The ILSP-CMA is a data-efficient and cost efficient technique which overcomes many of the problems associated with the Multi-target detection algorithms [6].

There are also some meta-heuristic techniques that provide the solution to the optimization problems by inspiring from the process of natural selection [10]. These are also called derivative-free methods like genetic algorithm (GA), Pattern Search (PS) and genetic algorithm hybridized with pattern search (GA-PS).All these are iterative processes. [11]

Areas where the statistics of signals and interference change continuously and are not known in advance, adaptive antenna arrays are used. Adaptive arrays consist of array of sensors whose weights are controlled by an adaptive processor which performs an optimization based on computations performed on the incoming signal. Adaptive antenna arrays are used in many practical applications these days.

Constant Modulus algorithms (CMA) have been used as an adaptive filtering technique for correcting multipath and interference degradations in constant envelope waveforms [8].Since multipath and interference causes unwanted amplitude modulation on the received signal and hence destroys constant envelope property of the signal. The key features of the study involve application of heuristic computing in directional of arrival estimation in a hybrid manner and in a mean square error sense.

The organization of this article is as follows: section 2 describes the system model of antenna array signal processing using uniform linear array. The details for derivative free methods have been narrated in section 3. Proposed methodology along with the hybrid derivative free method logical steps is provided in section 4 . In the section 5 , scenario based simulation and results along with their statistical analysis for the stability of the proposed scheme have been provided. Moreover the conclusions have been reveled in the last section along with some details for future work.

\section{SYSTEM MODEL}

Let the uniformly spaced linear array lying along the z-axis as shown in Fig.1, the steering vector can be computed as follows. Let the first antenna element is located at the reference location $\left(\mathrm{x}_{\mathrm{i}}, \mathrm{y}_{\mathrm{i}}, \mathrm{z}_{\mathrm{i}}\right)=(0,0,0)$. The incoming plane wave at element 2 travels a distance longer by an amount equal to 
$d \operatorname{Cos} \boldsymbol{\theta}$ with respect to the wave arriving at the first element, and hence arrives later.

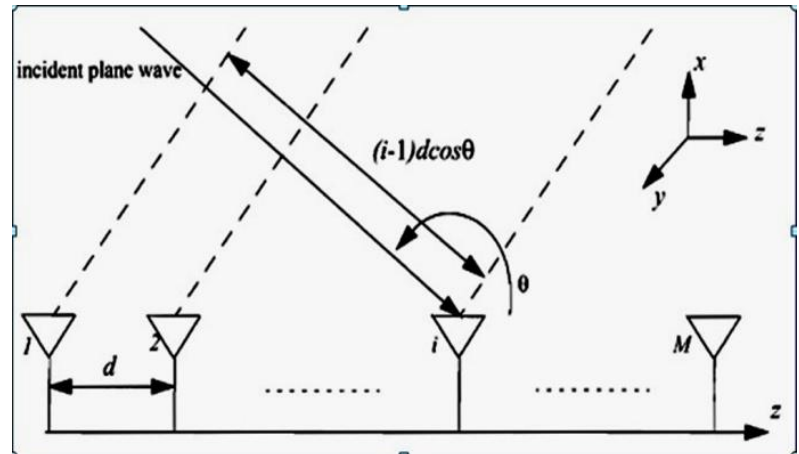

Fig. 1: The system model of Uniform Linear Array of antennas for DOE estimation

The basic relation for DOA estimation is provided below in the vector form along with the relation of the steering vector.

$$
X(k)=A(\Theta) S(k)+n(k)
$$

$$
a(\theta)=\left[\begin{array}{c}
1 \\
e^{j \beta d \operatorname{Cos} \theta} \\
\mathrm{M} \\
e^{j \beta(M-1) d \cos \theta}
\end{array}\right]
$$

\section{PROPOSED METHODOLOGY}

The derivative free methods based on the global search technique like genetic algorithm and local optimizer like pattern search has been provided along with the hybrid approach.

\subsection{Genetic Algorithm}

In GA population of solution candidates to an optimization problem is evolved towards better solution. Optimization is the mathematical programming in which we select the best element from some sets of available alternatives. Evolution comes due to iterative process. Repetition of process is called Iteration/Iterative process. Each solution candidate (Chromosome) can be mutated and altered. A population of randomly generated solution candidates is called Generation. In each generation the fitness of every solution candidate/individual is evaluated.GA terminates when satisfactory fitness level is achieved. Value of objective function is called "fitness".

Generation is the division of particles that differ by their number and mass but their interactions are identical. John Holland who invented this in early 1990's.GA is adaptive heuristic search algorithm based on evolutionary ideas of natural selection and genetics. It is better than conventional artificial intelligence. A GA is simple to implement but its behavior is difficult to understand.

John Holland observed the following things in nature before making the Genetic Algorithm.
- Individuals in population compete for resources and mates

- Individuals successful in each competition produce more offspring's than those individuals that perform poorly

- Genes from good individuals propagate throughout the population so that good parents produce offspring's that are better than the parents

- Thus successive generation is evaluated that is more suited to their environment.

\subsection{Pattern search}

A pattern is an image, usually small used for filling regions by placing copies of the pattern side by side like ceramic tiles A pattern is a set of vectors $\left\{v_{i}\right\}$ that the pattern search algorithm uses to determine the point to be searched at each iteration. The set $\left\{v_{i}\right\}$ is defined by the number of independent variables in the objective function, $N$, and the positive basis set.

Two commonly used positive basis sets in pattern search algorithms are the maximal basis, with $2 N$ vectors, and the minimal basis, with $N+1$ vectors. If the pattern search algorithm finds a point in the mesh that improves the objective function at the current point, the new point becomes the current point at the next step of the algorithm. A method used for solving optimization problems that does not require any information about the gradient of the objective function is called "Direct Search method" Many traditional optimization methods use information about the gradient or higher derivatives to search for an optimal point. A direct search algorithm searches a set of points around the current point, looking for one where the value of the objective function is lower than the value at the current point. We can use direct search to solve problems in which the objective function is not differentiable, or is not even continuous.

There are three direct search algorithms

- Generalized pattern search (GPS) algorithm

- $\quad$ Generating set search (GSS) algorithm

- Mesh adaptive search (MADS) algorithm

\subsection{Hybrid Approach (GA-PS)}

In the hybrid approach the following steps has been taken:

Step 1: Generate a random population of the chromosome length equal to the number of sources of the antennas.

Step 2: Formulate the fitness function based on DOA estimation for varying sources $\mathrm{G}(\mathbf{x})$.

Step 3: calculate the fitness value of the merit function based on mean square error (MSE).

Step 4: Rank the values of the fitness function in ascending order and perform the basic genetic operators like crossover, mutation and selection.

Step 5: The output of the global search genetic algorithm is provided as a start point to pattern search scheme.

Step 6: Store the results of the hybrid approach for 100 independent runs to see the convergence and robustness of the algorithm. 


\section{SIMULATION AND RESULTS}

The scenario based result and discussion is provided in this section by taking the cases of targets close to each other and targets away from each other and using different number of sources with varying antenna elements.

\subsection{Case I: Targets are away from each other}

The targets are away from each other while two, three, four and five sources have been taken into account at $10 \mathrm{db}$ noise provided in the simulations using white Gaussian noise. The parameter setting and values used for simulation have been provided in Table 1 in order to reproduce the results. By using these parameter values, the results can be observed the same although the method is stochastic.

Table 1. Parameter setting / values of GA and PS

\begin{tabular}{|c|c|c|c|}
\hline \multicolumn{2}{|c|}{ Genetic Algorithm } & \multicolumn{2}{c|}{ Pattern Search } \\
\hline Parameters & Setting & Parameters & Setting \\
\hline $\begin{array}{c}\text { Population } \\
\text { type }\end{array}$ & $\begin{array}{c}\text { Double } \\
\text { vector }\end{array}$ & Poll method & $\begin{array}{c}\text { GPS } \\
\text { Positive } \\
\text { basis } 2 \mathrm{~N}\end{array}$ \\
\hline $\begin{array}{c}\text { Population } \\
\text { size }\end{array}$ & 240 & Complete poll & Off \\
\hline $\begin{array}{c}\text { Initial range } \\
\text { Scaling } \\
\text { function }\end{array}$ & Rank & $\begin{array}{c}\text { Mesh initial } \\
\text { size }\end{array}$ & 1.0 \\
\hline $\begin{array}{c}\text { Selection } \\
\text { function }\end{array}$ & $\begin{array}{c}\text { Stochastic } \\
\text { uniform }\end{array}$ & $\begin{array}{c}\text { Expansion } \\
\text { factor }\end{array}$ & 2 \\
\hline Mutation & $\begin{array}{c}\text { Constraints } \\
\text { dependant }\end{array}$ & $\begin{array}{c}\text { Contraction } \\
\text { factor }\end{array}$ & 0.5 \\
\hline Crossover & Scattered & Initial penalty & 10 \\
\hline $\begin{array}{c}\text { Penalty } \\
\text { factor }\end{array}$ & 100 & Penalty factor & 100 \\
\hline Generations & 500 & Bind Toll & $1 \mathrm{e}-03$ \\
\hline $\begin{array}{c}\text { Stall } \\
\text { Generation }\end{array}$ & 50 & Max. Iterations & 500 \\
\hline Others & Default & Other & Default \\
\hline
\end{tabular}

The fitness values achieved by using GA, PS and GA-PS are provided in Fig.2 to Fig.5, respectively. It has been observed from the figure that fitness value achieved by hybrid approach is much better as compared to the pattern search and genetic algorithm individually. The values of the genetic algorithm are so close to pattern search which is not so visible for the two sources but for other cases the effects can be seen accordingly. It is also worth mentioning that the value of the fitness lies in the range $10^{-09}$ to $10^{-12}, 10^{-09}$ to $10^{-11}, 10^{-08}$ to $10^{-}$ 10 , and $10^{-06}$ to $10^{-8}$ for two, three, four and five sources respectively using the hybrid approach GA-PS. It is quite evident that the level of the accuracy decreases with the increase in the number of sources that is quite natural.

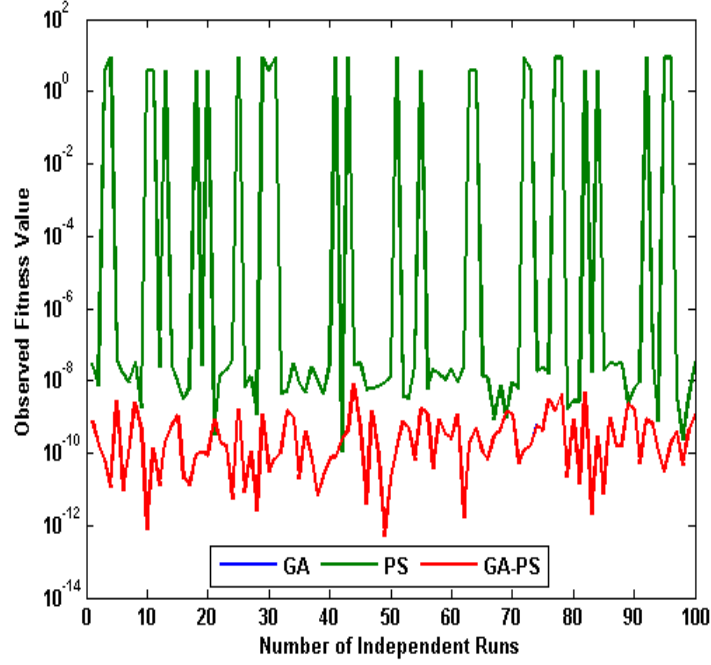

Fig2. Fitness achieved for two sources for using derivative free methods

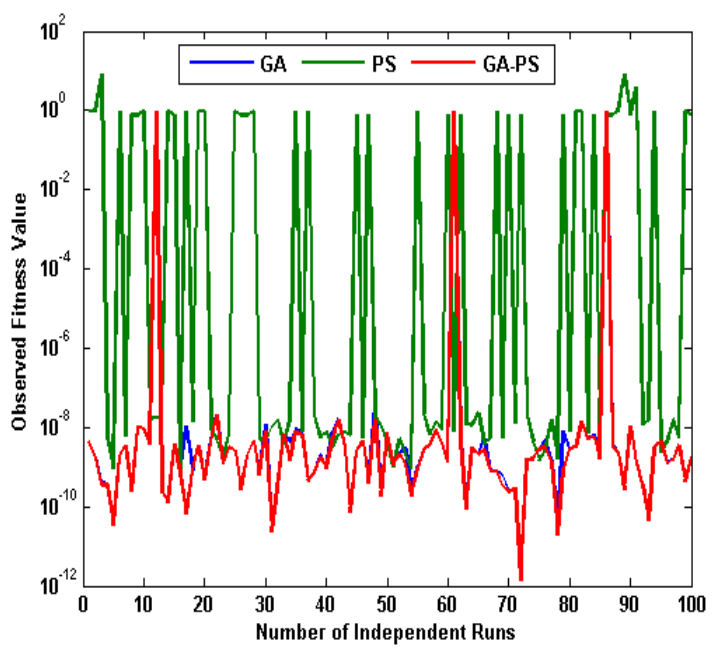

Fig3. Fitness achieved for three sources for using derivative free methods

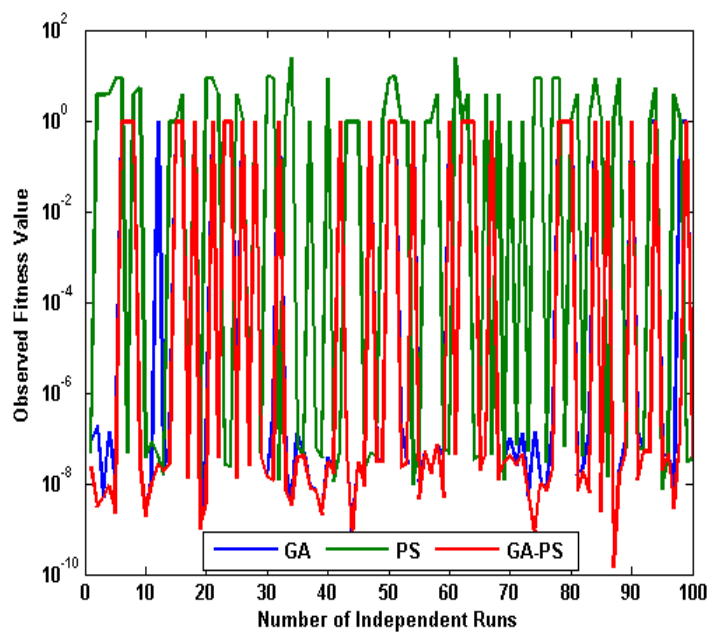

Fig4. Fitness achieved for four sources for using derivative free methods 


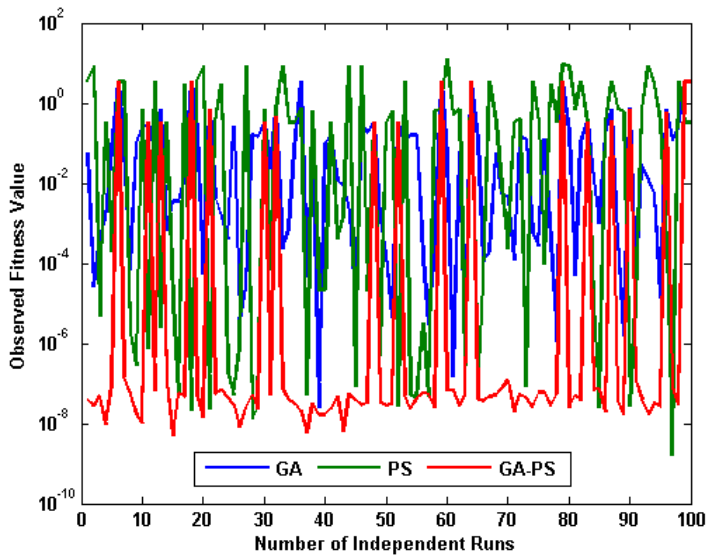

Fig5. Fitness achieved for five sources for using derivative free methods

Due to the stochastic nature of the derivative free methods, the 100 independent runs of the algorithms have been executed in order to see the reliability and convergence of the given schemes.

The computational budget is also taken in term of time complexity of the independent runs. The results are provided from Fig. 6 to Fig, 9.

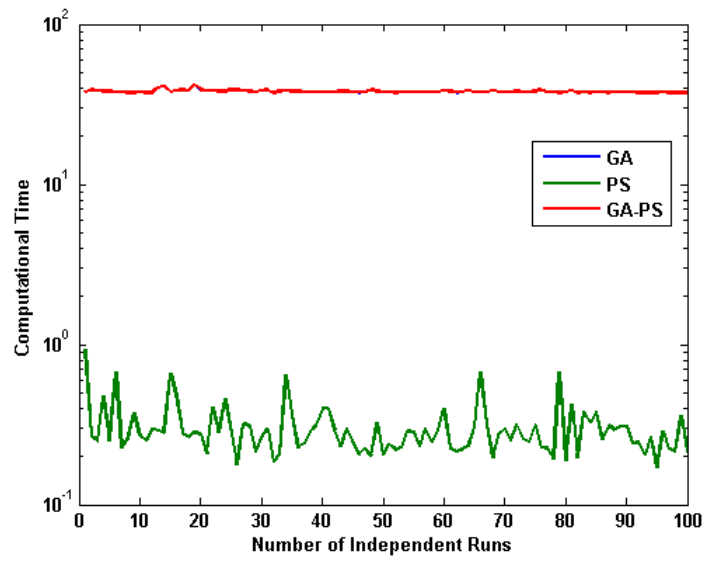

Fig6. Computational time needed for two sources using derivative free methods

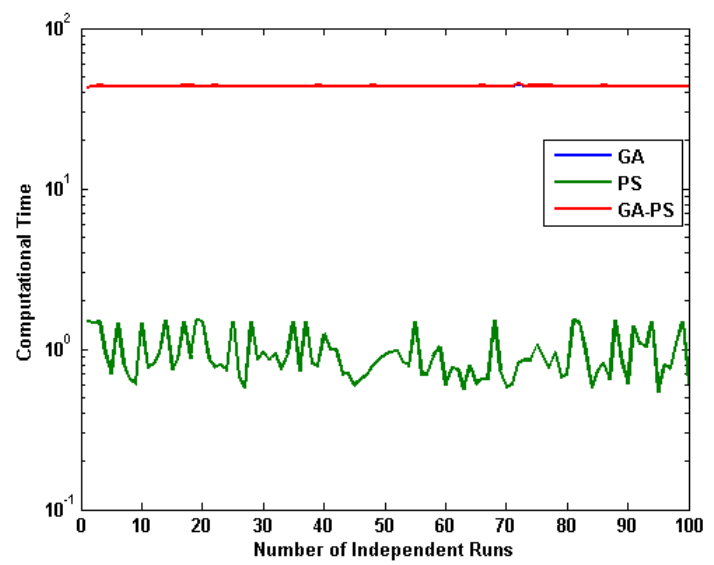

Fig7. Computational time needed for three sources using derivative free methods

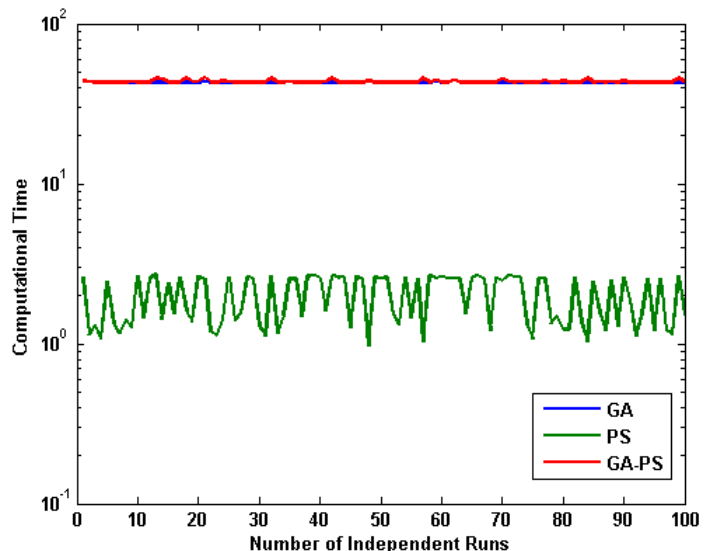

Fig8. Computational time needed for four sources using derivative free methods

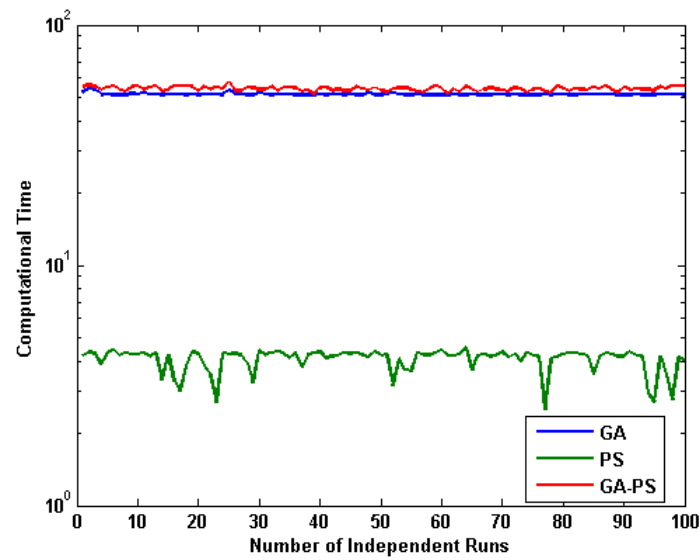

Fig9. Computational time needed for five sources using derivative free methods

\subsection{Case II: Targets are close to each other}

In this case the targets are very much close to each other and the measurements of such targets is difficult to measure. This case has also been simulated with varying sources. The fitness achieved along with the computational time required is provided below in Fig.10 to Fig. 15. Although the computational budget for hybrid approach is slightly high but is effect can be overhead due to high increase in the fitness achieved.

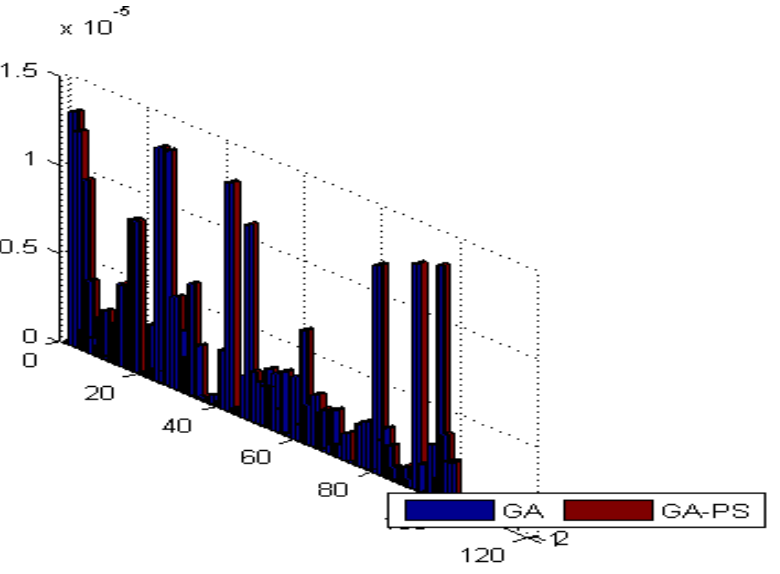

Fig10. Fitness achieved for two sources close to each other using hybrid scheme 


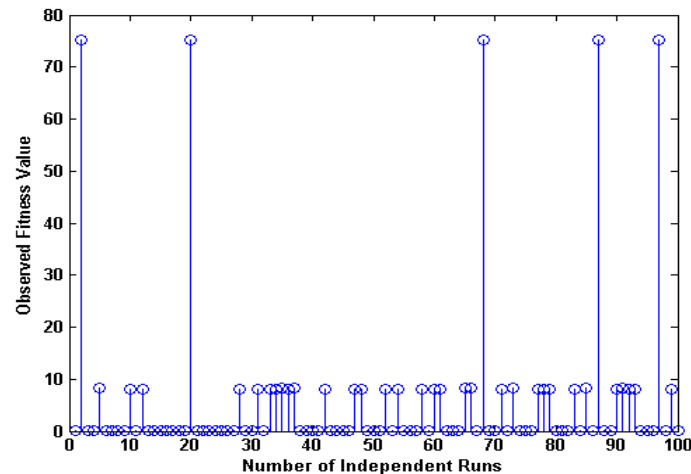

Fig11. Fitness achieved for three sources close to each other using hybrid scheme

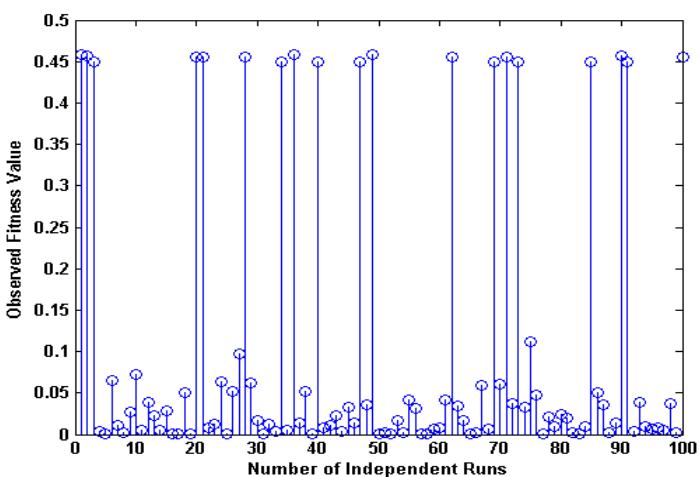

Fig12. Fitness achieved for four sources close to each other using hybrid scheme

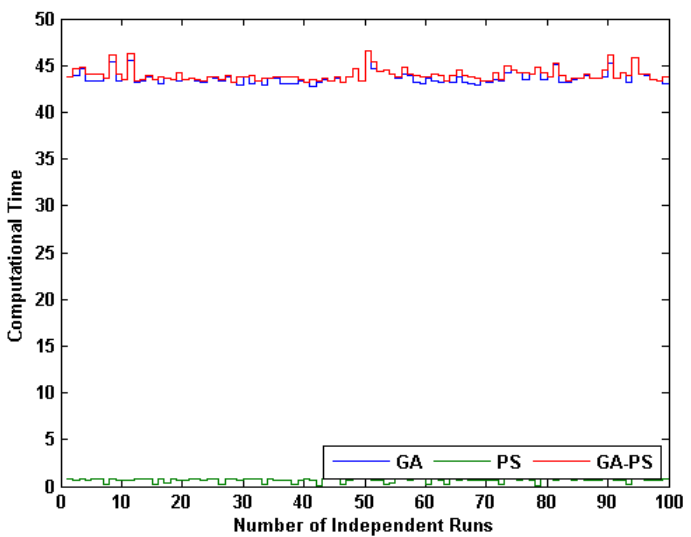

Fig13. Computational time needed for two close targets

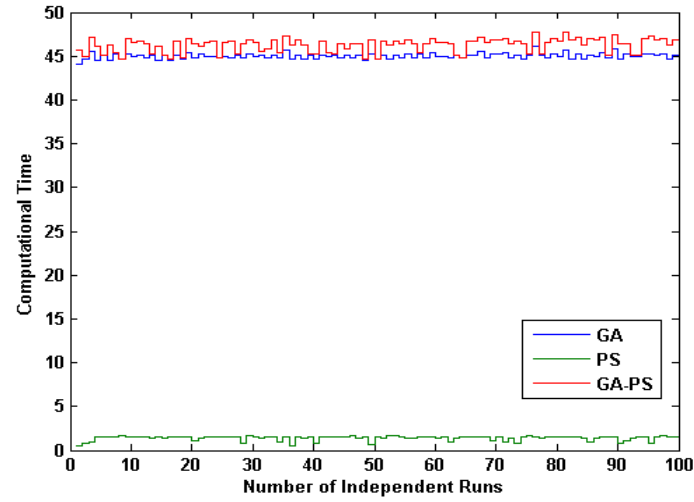

Fig14. Computational time needed for three close targets

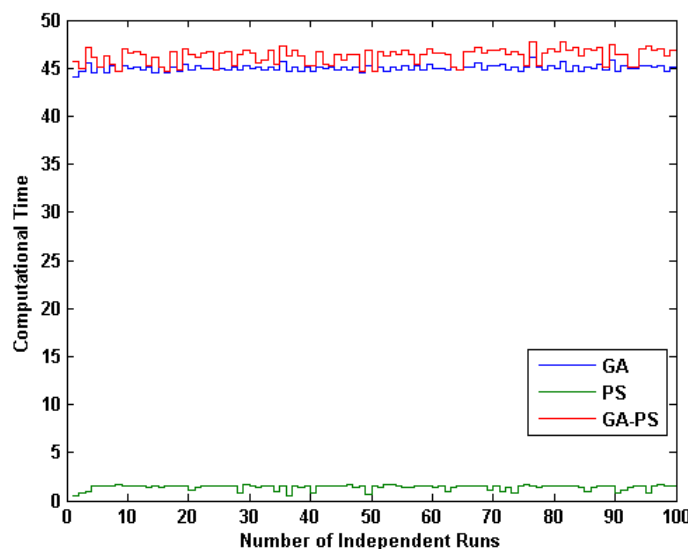

Fig15. Computational time needed for four close targets

\section{CONCLUSIONS}

Based upon the observation in the section of results and discussion following conclusions can be drawn.

- The derivative free methods are applicable to solve the radars simulation problem based on the targets estimation in the far field.

- The level of accuracy of the hybrid approach in both of the cases is much better than that of genetic algorithm and pattern search individually.

- The computational budget of hybrid approach is slightly high as compared to local and global search technique independently but fitness values have been incredibly improved.

- The convergence of 100 independent runs proves the validity and robustness of the derivative free methods for direction of arrival estimation in array of antennas.

- The proposed scheme is provides ease in implementation, simplicity of code and can easily installed on the hardware platform.

In future, one can work on the other geometry of antenna used in array signal processing like $\mathrm{L}$ shape and $2 \mathrm{~L}$ shape parametric estimation. However, the exploitation of other meta heuristic methods like ACO, Firefly method etc could a good area of future research.

\section{ACKNOWLEDGMENTS}

The corresponding author are really thankful for the support provided by the experts from University of engineering and Technology Peshawar

\section{REFERENCES}

[1] Haykin, S. "Radar array Processing for Angle-of-Arrival Estimation," Array Signal Processing,Prentice Hall, NJ, ch. 4, pp. 194-292, 1985

[2] R.O. Schmidt, "Multiple Emitter location and signalparameter estimation"in Proc. RADC SpectrumEsitmation Workshop, Griffiths, New York, pp.243-258,1979. Reprented in IEEE Transactions onAntennas and propagation, Vol.34, no.3,pp.276380,March 1986.

[3] A Singularly Valuable Decomposition: The SVD ofa Matrix By Dan Kalman The American UniversityWashington, DC 20016 on February 13, 2002. 
[4] F.Schweppe, "Sensor-array data processing forMultiple signal sources",IEEE Trans.Inf. Theory,vol.14, no.2, pp.294-305, 1968.

[5] M.Wax and T.Khilath, "Optimization localizationof multiple sources by passive arrays" IEEE Trans.Acoust., Speech, signal process,vol.31, no.5, pp1210-1217, 1983.

[6] Parra, I.P., Improved Constant ModulusAlgorithm for Smart Antenna Systems, MastersThesis, The University of Texas at Austin, May1995.

[7] MUSIC Algorithm by Brian L. Evans, 211-105Cory Hall, Berkeley, CA 94720-1772

[8] Triechler,J.R.,Agee, B.G., A new approach tomultipath Correction of constant modulus signals,"IEEE transaction on Acustics, Speech and signalprocessing,vol. ASSP-31 ,no.2 April 1983.

[9] J. Capon, "High Resolution frequency-wavenumber spectrum analysis," Proc. IEEE, vol. 57,pp.1408-1418, Aug. 1969.

[10] Eiben, A. E. et al (1994). "Genetic algorithmswith multiparent recombination". PPSN III:Proceedings of the International Conference onEvolutionary Computation. The Third Conference onParallel Problem Solving from Nature: 78-87. ISBN3-540- 58484-6

[11] F. Zaman1, *, I. M. Qureshi2, A. Naveed3, J. A.Khan1, and R. M. A. Zahoor1 "amplitude anddirectional of arrival es- timation: comparisonbetween different techniques" Progress InElectromagnetics Research B, Vol. 39, 319-335, 2012 